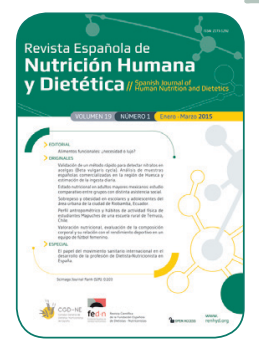

\title{
ORIGINAL
}

\section{Valoración nutricional, evaluación de la composición corporal y su relación con el rendimiento deportivo en un equipo de fútbol femenino}

\author{
María González-Neira ${ }^{a}$, Ismael San Mauro-Martín ${ }^{a, *}$, Belén García-Angulo ${ }^{a}$, Diana Fajardo ${ }^{a}$, Elena \\ Garicano-Vilar $^{\text {a }}$
}

a Centros de Investigación en Nutrición y Salud, España.

* research@grupocinusa.com

Recibido el 26 de mayo de 2014; aceptado el 16 de septiembre de 2014 .

PALABRAS CLAVE

Fútbol;

Rendimiento Deportivo;

Nutrición Deportiva.
Valoración nutricional, evaluación de la composición corporal y su relación con el rendimiento

deportivo en un equipo de fútbol femenino

\section{RESUMEN}

Introducción: En el fútbol femenino, cumplir con los requerimientos nutricionales podría influir positivamente en el rendimiento deportivo. El objetivo del presente estudio fue analizar la ingesta nutricional y la composición corporal (CC), comprobando su relación con el rendimiento deportivo.

Material y métodos: Estudio observacional, descriptivo, prospectivo y correlacional, realizado en 17 jugadoras del equipo semiprofesional de Torrelodones C.F. de Madrid. Se determinó el peso y la talla y se calculó el índice de masa corporal (IMC). La CC se determinó mediante bioimpedancia eléctrica (BIA). La ingesta nutricional se analizó a través de un registro dietético semanal (valorado a través del programa DIAL) y el test KIDMED. Se determinó el volumen máximo de oxígeno $\left(\mathrm{VO}_{2 \text { máx }}\right)$ a partir de la prueba Course-Navette y se realizó el test de percepción subjetiva de esfuerzo (PSE).

Resultados: IMC: $22,80 \pm 2,9 \mathrm{~kg} / \mathrm{m}^{2}$; masa grasa: $24,51 \pm 5,5 \%$; y masa magra: $43,6 \pm 2,9 \mathrm{~kg}$. La ingesta energética fue de $1901 \pm 388 \mathrm{kcal}$. El aporte de hidratos de carbono supuso el 40,3 $\pm 5,6 \%$, las proteínas el $15,9 \pm 3,29 \%$ y las grasas el $41,8 \pm 4,1 \%$ de las kcal totales. Existió una relación inversa entre IMC y peso, y los valores de $\mathrm{VO}_{2 \text { máx. }}$ Hubo una pérdida de peso significativa después del entrenamiento $(p=0,003)$ y del partido $(p=0,008)$.

Conclusiones: La alimentación fue inadecuada en las jugadoras, no correspondiendo la ingesta de nutrientes con sus requerimientos, a pesar de entrenar en un equipo semiprofesional. Debido a la importancia que la nutrición juega en la competición y rendimiento deportivo, se debe seguir trabajando para una recomendación adecuada en este colectivo. 


\section{KEYWORDS}

Soccer;

Athletic

Performance;

Sports Nutritional

Science.

Nutritional and body composition assessment and its relationship with athletic performance in a women's soccer team

\section{ABSTRACT}

Introduction: In women's soccer, meeting the nutritional requirements could have a positive impact on athletic performance. The aim of this study was to analyze dietary intake and body composition (CC), proving their relationship with athletic performance.

Material and Methods: Observational, descriptive, prospective, and correlational study performed on 17 players from Torrelodones C.F., a semi-professional team in Madrid. Weight and height were measured and the body mass index (IMC) was calculated. The CC was determined by bioelectrical impedance analysis (BIA). Dietary intake was analyzed through a weekly food registry (assessed with the program DIAL) and the KIDMED test. The maximum volume of oxygen $\left(\mathrm{VO}_{2 \text { max }}\right)$ was determined by the Course-Navette test and the rating of perceived effort test (PSE) was also taken.

Results: IMC was $22.80 \pm 2.9 \mathrm{~kg} / \mathrm{m}^{2}$, fat mass was $24.51 \pm 5.5 \%$ and lean mass $43.6 \pm 2.9 \mathrm{~kg}$. Energy intake was $1901 \pm 388 \mathrm{kcal}$. The contribution of carbohydrates accounted for $40.3 \pm 5.6 \%$, protein $15.9 \pm 3.29 \%$, and fat $41.8 \pm 4.1 \%$ of total kcal. There was an inverse relationship between IMC and weight, and $\mathrm{VO}_{2 \max }$ values. There was a significant weight loss after training $(p=0.003)$ and after the match $(p=0.008)$.

Conclusions: Player's diet was inadequate, not corresponding nutrient intake with their requirements, despite training in a semi-professional team. Because of the importance that nutrition plays in competition and athletic performance, investigations should continue working on an appropriate recommendation for this collective.

\section{CITA}

González-Neira M, San Mauro-Martín I, García-Angulo B, Fajardo D, Garicano-Vilar E. Valoración nutricional, evaluación de la composición corporal y su relación con el rendimiento deportivo en un equipo de fútbol femenino. Rev Esp Nutr Hum Diet. 2015; 19(1): 36 - 48. DOI: 10.14306/renhyd.19.1.109

\section{INTRODUCCIÓN}

Desde hace más de treinta años, el fútbol femenino forma parte de las disciplinas deportivas ${ }^{1}$. El fútbol es un deporte técnico-táctico en equipo, de resistencia y a la vez de contacto, englobando actividades de baja, media y alta intensidad (con momentos anaeróbicos y otros aeróbicos). Además de la influencia que ejercen la genética y el entrenamiento individual en el rendimiento deportivo, podemos considerar la dieta del deportista como uno de los factores más importantes a la hora de optimizar dicho rendimiento ${ }^{2}$. Por ello, cumplir los requerimientos de ingesta energética y nutricional influirá positivamente en el rendimiento, tanto físico como deportivo de los futbolistas ${ }^{3-5}$. Una nutrición apropiada ayuda a optimizar la producción de energía durante el ejercicio. Además una nutrición no apropiada en el deportista, puede contribuir, entre otras, a la aparición de lesiones deportivas².
Los avances en la fisiología del ejercicio han hecho posible ir concretando algunas recomendaciones específicas para cubrir las necesidades nutricionales de los atletas, es decir, conocer prácticas dietéticas que ayuden a los atletas a optimizar su rendimiento deportivo².

En cuanto al género, el gasto de energía medio aproximado durante un partido es de $1.100 \mathrm{kcal}$ para una futbolista de $60 \mathrm{~kg}^{6}$, menor que en hombres. En relación a las necesidades de nutrientes del futbolista, destacar que es importante mantener un consumo adecuado de hidratos de carbono (HC) (55-60\%), ya que cuanto más glucógeno y glucosa haya disponibles para el músculo, más rápido y durante más tiempo podrán correr los jugadores ${ }^{6,7}$. Las proteínas parecen desempeñar una función importante en la recuperación muscular post-esfuerzo, sugiriendo incrementar las necesidades diarias hasta un máximo de 1,2 a $1,6 \mathrm{~g} / \mathrm{kg}$ de peso corporal $(P C)^{6}$. Las grasas se aconseja que supongan entre un $25-30 \%$ de la energía total de la 
dieta, con un contenido alto de ácidos grasos insaturados, disminuyendo el porcentaje de forma evidente en los momentos previos a la competición y llegando a ser nulo durante la misma ${ }^{8,9}$. Dichas necesidades son similares a las que se presentan en un patrón de Dieta Mediterránea. Aunque llevar una alimentación correcta no es el único factor que vaya a condicionar el éxito de un jugador, si ésta se descuida el deportista podría ver su rendimiento físico mermado ${ }^{10}$.

Durante el período de formación de los jóvenes futbolistas tienen lugar una serie de cambios antropométricos que determinarán el estado final del futbolista en cuanto a estado de forma física y potencial de rendimiento deportivo. Por lo que la nutrición durante esta etapa de desarrollo puede ser decisiva para el futuro deportista?

Además de la alimentación, la composición corporal (CC) y el PC son dos de los muchos factores que favorecen un óptimo rendimiento deportivo ${ }^{11}$. La presencia de grasa corporal (GC) en el deportista es necesaria, ya que un bajo porcentaje se asocia con un deterioro de la salud y del rendimiento deportivo ${ }^{12}$. Son diversos los estudios que llegan a la conclusión de que, por término medio, las mujeres futbolistas presentan en torno a un $21 \%$ de masa grasa $(M G)^{13}$. Aun así, considerando el tamaño del campo y el tiempo de juego, se debe tener en cuenta que la alta cantidad de grasa y una robustez elevada perjudican el desempeño físico. En un juego de 90 minutos se recorren cerca de 10 kilómetros a una frecuencia cardiaca máxima entre el 80 y 90\%, lo cual resulta difícil para deportistas con exceso de peso ${ }^{14}$. Fundamento de eso es el trabajo de Mladenovic en $2005^{15}$, que estudió los cambios ocurridos en los equipos femeninos de Serbia y Montenegro, mostrando que al disminuir el índice de masa corporal (IMC) en un $3,75 \%$ y la grasa en un $1,7 \%$, se puede aumentar en un $18,4 \%$ el
$\mathrm{VO}_{2 \text { máx }}{ }^{15-17}$. No obstante, los niveles óptimos de grasa que permiten un buen rendimiento deportivo deberían de valorarse individualmente en cada caso.

Maximizar la masa muscular (MM) es deseable en deportes que requieran fuerza, potencia y resistencia muscular, pero puede suponer un inconveniente en deportes de resistencia ${ }^{13,18}$ En los deportes de equipo en general, y fútbol en particular, es más difícil que en los deportes individuales describir un morfotipo ideal por la presencia de distintas posiciones de juego y de distintas funciones sobre el terreno de juego ${ }^{19}$. A pesar de ello, se han publicado valores en fútbol femenino que se resumen en la Tabla $1^{20}$, siendo las futbolistas consideradas endomorfas, mesomorfas y endomesomorfas ${ }^{21}$.

En esta tabla se observa que los grupos evaluados son diferentes en su somatotipo, evidenciando biotipos diferentes. Quizás a esta edad, donde la función de juego y el tiempo de entrenamiento no parecen causar modificaciones morfológicas en estas deportistas, el somatotipo no es factor discriminante de rendimiento deportivo ${ }^{18}$.

Los objetivos del presente estudio fueron analizar la ingesta nutricional y la CC y su relación con el rendimiento deportivo de un equipo de fútbol semiprofesional femenino de la Comunidad de Madrid.

\section{MATERIAL Y MÉTODOS}

La población de estudio estuvo constituida por 17 mujeres de entre 16 y 37 años, todas ellas jugadoras del equipo semiprofesional femenino de Torrelodones C.F., perteneciente a la Segunda División Femenina de España, población

Tabla 1. Comparación de Somatotipo con otros autores: somatotipo medio, puntos de la somatocarta y distancia de dispersión del somatotipo medio (SDD del SM).

\begin{tabular}{|c|c|c|c|c|c|c|c|c|c|}
\hline Autor/Año & $\mathbf{n}$ & Especialidad & Nivel & ENDO & MESO & ECTO & $\mathbf{x}$ & $\mathbf{Y}$ & SDD del SM \\
\hline Sub 17 Sudamérica & 28 & Fútbol & Selecciones Sudamérica U17 & 4,23 & 3,77 & 2,04 & $-2,2$ & 1,3 & \\
\hline Almagià et al. (2008) & 25 & Fútbol & Selección chilena U20 & 3,9 & 4,7 & 1,5 & $-2,4$ & 4 & 2,8 \\
\hline Can et al. (2004) & 17 & Fútbol & Profesional turco (3 años) & 3,07 & 3,55 & 2,43 & $-0,64$ & 1,6 & 2,7 \\
\hline
\end{tabular}

n: frecuencia; Endo: endomorfia; Meso: mesomorfia; Ecto: ectomorfia. 
donde fueron tomados los datos. Se trató de un estudio observacional, descriptivo, prospectivo y correlacional en el que se realizó un cuestionario ad hoc (datos personales, anamnesis clínica, hábitos de vida, alimentación, deporte, etc.), en marzo del año 2013. Se midieron el peso y la talla, y se calcularon el IMC, el porcentaje de grasa, la masa libre de grasa $(\mathrm{kg})$ y el porcentaje de agua de cada jugadora. La CC se determinó mediante bioimpedancia eléctrica (BIA) tetrapolar monofrecuencia $(50 \mathrm{kHz})$, de la compañía TANITA, Modelo BP-601. Se estandarizó un protocolo para la medición de la CC mediante la BIA que consistió en: 1) ayuno previo de 3 horas como mínimo; 2) haber orinado antes de realizar la medición; 3) no haber ingerido café, té o mate, ni haber realizado actividad física desde 12 horas antes de tomar la medición; 4) no haber ingerido bebidas alcohólicas ni diuréticos desde 24 horas antes de realizar la impedancia.

La estatura, definida como la distancia entre el vertex (vértice craneal) y el plano de sustentación -también denominada como talla en bipedestación o simplemente talla- se midió con un estadiómetro Seca 216, rango 3,5 a 230 cm, con una precisión de $1 \mathrm{~mm}$. La medición se realizó con el sujeto de pie, sin zapatos, completamente estirado, colocando los pies paralelos y con los talones unidos (apoyados en el borde posterior) y las puntas ligeramente separadas (formando aproximadamente un ángulo de $60^{\circ}$ ), las nalgas, hombros y cabeza en contacto con un plano vertical. La cabeza se mantuvo cómodamente erguida con el borde orbitario inferior en el mismo plano horizontal que el conducto auditivo externo (plano de Frankfurt). El antropometrista realizó una tracción a nivel de los procesos mastoideos, para facilitar la extensión completa de la columna vertebral. Los brazos colgaban a lo largo del cuerpo de una manera natural con las palmas de las manos frente a los muslos. Se le pidió al sujeto que realizara una inspiración profunda para obtener la extensión máxima de la columna. Se descendió lentamente la plataforma horizontal del estadiómetro hasta contactar con la cabeza del estudiado, ejerciendo una suave presión para minimizar el efecto del pelo. Los ojos del examinador estaban por lo menos a la misma altura del sitio donde el panel movible hace contacto con la cabeza.

Se analizó la ingesta nutricional y los hábitos nutricionales a través de un registro dietético semanal y el test KIDMED ${ }^{22}$. Una vez finalizado, se realizó la valoración nutricional de la alimentación de las deportistas mediante el programa informático DIAL ${ }^{23}$. En el cuestionario ad hoc, de 2 folios de extensión, se incluyeron ítems sobre hábitos dietéticos (número de tomas, realización de comidas fuera de casa, presencia de picoteo, percepción subjetiva de la calidad de su alimentación, etc.).

Se realizó una prueba de rendimiento físico -test de Course-
Navette $(\mathrm{CN})^{24}$-, para determinar el volumen de oxígeno máximo $\left(\mathrm{VO}_{2 \text { máx }}\right)$, un día de partido y otro de entrenamiento. En ambos días se midió el PC y el porcentaje de agua, antes y después del ejercicio, y se incluyó el test de percepción subjetiva de esfuerzo (PSE), basado en la escala de Borg ${ }^{25,26}$.

El requerimiento calórico en entrenamiento se determinó a partir de la fórmula de Cunningham ${ }^{27}$ (tasa metabólica en basal $=500+22 \times$ (masa corporal magra en $\mathrm{kg})$ ), multiplicada por un factor de actividad individual ${ }^{28}$.

El requerimiento de proteínas se calculó de manera individual, obteniendo la media y teniendo en cuenta los requerimientos específicos para jugadores de fútbol $\left(1,2-1,6 \mathrm{~g} / \mathrm{kg}\right.$ de PC/día) ${ }^{6}$.

Conociendo la ingesta recomendada de energía (IRE), el requerimiento de glúcidos se calculó de manera individual y teniendo en cuenta la energía total requerida y las características del ejercicio físico (kcal/día de $\mathrm{HC}=\mathrm{IRE} \times \mathrm{x} \%$ recomendado $\mathrm{HC}$; g de $\mathrm{HC}=\mathrm{kcal} /$ día de $\mathrm{HC} \times 4 \mathrm{kcal}$ (porg de $\mathrm{HC}$ ). Conociendo los requerimientos de $\mathrm{HC}$ de cada jugadora, se valoró si éstas ingerían las cantidades adecuadas o no en base a los datos de ingesta extraídos a partir del registro dietético de una semana, haciendo la media de los 7 días.

Previo al estudio, se realizó una sesión informativa con las jugadoras y los entrenadores, para evitar cualquier duda en la cumplimentación de los registros o cuestionarios. Todas las participantes fueron informadas, de forma escrita y oral, sobre la finalidad del estudio y firmaron una hoja de consentimiento informado. Todos los datos fueron recogidos por dietistas-nutricionistas entrenados y formados, homogenizando con un protocolo de recogida de datos y monitorización del estudio.

EI análisis de los datos se realizó con el software SPSS, versión 21. Se analizaron descriptivamente las variables de medida, comprobando el ajuste de las variables numéricas a la distribución normal mediante la prueba de ShapiroWilk. Posteriormente se aplicó la Prueba $T$ de Student para analizar la diferencia de peso y porcentaje de agua tras los entrenamientos y partidos para dos muestras relacionadas o la correspondiente prueba no paramétrica de rangos con signo de Wilcoxon, cuando la distribución no fue normal. En los valores de ingesta nutricional se utilizaron estas pruebas para comprobar la diferencia entre el consumo y el requerimiento de kcal, proteína y glúcidos. Para estudiar la relación del rendimiento deportivo con la CC, la ingesta nutricional y otros factores relacionados, se utilizó el coeficiente de correlación de Pearson de asociación lineal, cuando se trataba de variables numéricas. Para el análisis de la relación con variables dicotómicas se aplicó la Prueba T de Student para dos muestras relacionadas o la 
correspondiente prueba no paramétrica de rangos con signo de Wilcoxon. También se utilizó la prueba no paramétrica de Kruskal-Wallis para el análisis de la relación del rendimiento deportivo con las variables categóricas cuando los grupos comparados no fueron homogéneos, usando el nivel de significación del $5 \%$.

\section{RESULTADOS}

El peso, la talla, el IMC y la CC (\% de MG y MM en $\mathrm{kg}$ ), se muestran en la Tabla 2. El peso medio de las jugadoras fue de $61,4 \pm 8,7 \mathrm{~kg}$. La talla media, según posiciones de juego, fue de $164,2 \mathrm{~cm}$ en las centrales; $161,3 \mathrm{~cm}$ en las delanteras; $161,5 \mathrm{~cm}$ en las laterales; y $164,6 \mathrm{~cm}$ en las medio-centros, destacando la talla de la portera que estuvo por encima de la media, en comparación con sus demás compañeras $(172 \mathrm{~cm})$. Los valores medios de IMC fueron de $22,8 \pm 2,9 \mathrm{~kg} / \mathrm{m}^{2}$. El porcentaje medio de $M G$ fue de $24,5 \pm 5,5 \%$ y la $M M$ de $43,6 \pm 2,9 \mathrm{~kg}$.

La ingesta media de energía, de nutrientes y el perfil calórico de la dieta de las jugadoras se muestra en la Tabla 3. Las ingestas de calorías semanal $(1.900,81 \pm 388,16 \mathrm{kcal})$ y de un día de entrenamiento $(1.931,93 \pm 535,77 \mathrm{kcal})$ fueron inferiores a las recomendadas $(2.592,35 \pm 264,01 \mathrm{kcal}$ ) (Figura 1). El perfil calórico medio estuvo constituido por un $40,3 \pm 5,7 \%$

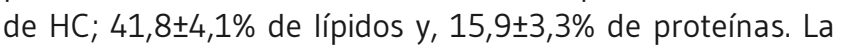
ingesta de $\mathrm{HC}$ simples fue de $87,9 \pm 26,9 \mathrm{~g}$. El consumo de HC horas previas ( 6 horas antes) a la práctica deportiva era inadecuado en el $50 \%$ de las jugadoras el día del entrenamiento, en el que se realizó la prueba de $\mathrm{CN}$, y en el 31,2\%, el día del partido. Presentaban un perfil lipídico con un alto consumo de ácidos grasos saturados (AGS) $(12,4 \pm 2,3 \%)$ y de ácidos grasos monoinsaturados (AGM) $(21 \pm 2,4 \%)$ y, bajo de ácidos grasos poliinsaturados (AGP) $(5,4 \pm 1,0 \%)$. La ingesta media de hierro semanal fue de $12,46 \pm 3,12 \mathrm{mg} /$ día.

Los resultados obtenidos a través del cuestionario KIDMED mostraron patrones de hábitos alimentarios poco saludables. Según los datos recogidos, el $41 \%$ de las jugadoras no consumía ninguna ración de fruta y de las que la consumían, sólo un $47 \%$ tomaba una segunda ración, el mismo porcentaje que tomaba al menos una ración de verdura, pescado 2-3 veces por semana y pasta y/o arroz 5 o más veces por semana. Las legumbres eran consumidas más de una vez a la semana por el 71\% y los frutos secos 2-3 veces por semana por el 29\%. La ingesta de 2 yogures y/o $40 \mathrm{~g}$ de queso al día sólo era realizado por un $56 \%$ de las futbolistas. El desayuno era omitido por un $12 \%$ de las encuestadas y, del $88 \%$ que sí lo realizaba, el $88 \%$ incluía al menos un lácteo, un $29 \%$ optaba por la bollería industrial y el $65 \%$ dijo elegir un cereal o derivado. Es destacable que un $18 \%$ de las jugadoras no usaba habitualmente el aceite de oliva en su casa, el mismo porcentaje que dijo consumir chucherías y caramelos varias veces al día. Por último, a través de este cuestionario, se obtuvo que el 59\% de las jugadoras comía una o más veces por semana en un centro de comida rápida. Sólo el 5,9\% obtuvo una calidad de dieta óptima según los valores correspondientes a la escala KIDMED de evaluación del ajuste a los estándares de la Dieta Mediterránea (adherencia a Dieta Mediterránea). Sin embargo, al preguntarles por su alimentación de forma subjetiva, el 64,7\% contestaron que "sí llevaban una alimentación adecuada", lo que difiere de la realidad tal y como se ha descrito, además de que el $76,5 \%$ afirmó que realizaba la conducta de picoteo.

Tabla 2. Estadísticos descriptivos de los parámetros antropométricos y composición corporal.

\begin{tabular}{l|c|c|c|c|c|c|c} 
Medida & $\mathbf{N}$ & $\mathbf{M}$ & Mn & Dt & Mín & Máx & SW \\
\hline Edad (años) & 17 & 22,12 & 21,00 & 4,55 & 16,00 & 37,00 & 0,001 \\
Peso (kg) & 17 & 61,51 & 60,60 & 7,92 & 50,30 & 79,80 & 0,010 \\
Estatura (cm) & 17 & 165,18 & 163,00 & 6,75 & 159,00 & 188,00 & 0,000 \\
IMC (kg/m²) & 17 & 22,81 & 21,70 & 2,96 & 19,80 & 29,90 & 0,002 \\
Grasa (\%) & 17 & 24,51 & 23,10 & 5,58 & 16,30 & 36,90 & 0,386 \\
Masa muscular (kg) & 17 & 43,64 & 43,30 & 2,99 & 38,00 & 50,00 & 0,717
\end{tabular}


Rev Esp Nutr Hum Diet. 2015; 19(1): 36 - 48

Valoración nutricional, evaluación de la composición corporal y su relación con el rendimiento deportivo en un equipo de fútbol femenino

Tabla 3. Valores de ingesta alimentaria.

\begin{tabular}{|c|c|c|c|c|c|c|c|}
\hline Medida & $\mathbf{N}$ & M & Mn & Dt & Mín & Máx & SW \\
\hline \multicolumn{8}{|l|}{ DATOS GENERALES E INGESTA CALÓRICA } \\
\hline Número de tomas & 17 & 3,94 & 4,00 & 1,03 & 2,00 & 5,00 & 0,011 \\
\hline KidMed & 17 & 5,35 & 6,00 & 2,00 & 1,00 & 8,00 & 0,070 \\
\hline Consumo semanal medio (Kcal) & 16 & 1900,81 & 2013,00 & 388,16 & 1133,00 & 2362,00 & 0,093 \\
\hline Ingesta energética el día de entrenamiento (Kcal) & 16 & 1931,94 & 2028,50 & 535,77 & 906,00 & 2926,00 & 0,504 \\
\hline Calorías procedentes de glúcidos simples & 16 & 351,63 & 317,50 & 107,78 & 181,00 & 540,00 & 0,294 \\
\hline Requerimiento calórico (entrenamiento) & 17 & 2592,35 & 2505,00 & 264,01 & 2243,00 & 3115,00 & 0,240 \\
\hline \multicolumn{8}{|l|}{ INGESTA DE PROTEÍNA } \\
\hline Proteína media semanal (\%) & 16 & 15,90 & 15,00 & 3,30 & 12,40 & 24,30 & 0,025 \\
\hline Proteína media semanal (gr) & 16 & 73,76 & 73,30 & 11,83 & 54,20 & 104,00 & 0,565 \\
\hline Requerimiento de proteínas en población adulta (gr) & 17 & 49,21 & 48,48 & 6,33 & 40,24 & 63,84 & 0,018 \\
\hline Requerimiento de proteínas en jugadores de fútbol (gr) & 17 & 86,12 & 84,84 & 11,08 & 70,42 & 111,72 & 0,018 \\
\hline \multicolumn{8}{|l|}{ INGESTA DE LÍPIDOS } \\
\hline Grasa media semanal (\%) & 16 & 41,85 & 41,40 & 4,12 & 35,10 & 49,10 & 0,677 \\
\hline Grasa media (gr) & 16 & 89,39 & 94,50 & 20,38 & 48,10 & 119,00 & 0,316 \\
\hline Grasas saturadas (\%) & 16 & 12,42 & 12,85 & 2,36 & 8,10 & 16,40 & 0,695 \\
\hline Grasas poliinsaturadas (\%) & 16 & 5,43 & 4,95 & 1,07 & 4,20 & 7,70 & 0,010 \\
\hline Grasas monoinsaturadas (\%) & 16 & 21,01 & 21,05 & 2,46 & 16,20 & 26,00 & 0,834 \\
\hline \multicolumn{8}{|l|}{ INGESTA DE GLÚCIDOS } \\
\hline Glúcidos (\%) & 16 & 40,34 & 41,25 & 5,67 & 27,90 & 52,20 & 0,504 \\
\hline Glúcidos (gr) & 16 & 182,78 & 187,00 & 52,26 & 66,40 & 266,00 & 0,934 \\
\hline Glúcidos simples (gr) & 16 & 87,98 & 79,40 & 26,90 & 45,30 & 135,00 & 0,296 \\
\hline Requerimiento glúcidos (gr) & 17 & 388,47 & 375,00 & 39,74 & 336,00 & 467,00 & 0,238 \\
\hline Ingesta de glúcidos 6 h antes del ejercicio (gr) & 16 & 86,94 & 91,55 & 49,09 & 2,60 & 163,50 & 0,758 \\
\hline Energía de glúcidos simples (\%) & 16 & 18,41 & 18,09 & 3,57 & 13,27 & 27,19 & 0,451 \\
\hline Glúcidos el día de entrenamiento (gr) & 16 & 158,63 & 167,95 & 56,16 & 46,80 & 245,00 & 0,429 \\
\hline \multicolumn{8}{|l|}{ OTRAS INGESTAS } \\
\hline Agua (I/día) & 16 & 1,40 & 1,37 & 0,46 & 0,67 & 2,54 & 0,241 \\
\hline Colesterol (mg/día) & 16 & 342,63 & 316,50 & 128,03 & 209,00 & 745,00 & 0,002 \\
\hline Hierro (mg/día) & 16 & 12,46 & 11,85 & 3,13 & 8,90 & 21,40 & 0,025 \\
\hline
\end{tabular}

N: frecuencia absoluta; M: media; Mn: mediana; Dt: desviación típica; Mín: valor mínimo; Máx: valor máximo; SW: valor crítico de significación correspondiente al estadístico de contraste de la prueba de normalidad de Shapiro-Wilk 
La pérdida de peso producida el día del entrenamiento (Test CN) es de $0,37 \pm 0,28 \mathrm{~kg}$ y el día del partido de $0,7 \pm 0,45 \mathrm{~kg}$, supusieron respectivamente una pérdida del $0,6 \pm 3,2 \%$ y del 1,1 $\pm 5,2 \%$ del PC (Tabla 4). El análisis de la normalidad de la variable peso, antes y después del evento, indicaron que el peso no siguió la distribución normal antes $(p=0,010)$ y después del entrenamiento $(p=0,007)$, así como antes $(p=0,011)$ y después del partido $(p=0,010)$. La diferencia relacionada del peso antes y después del entrenamiento arrojaron un resultado estadísticamente significativo $(z=-2,950$; $p=0,003)$, indicando que existe una pérdida de peso significativa asociada a la realización del entrenamiento. La pérdida de peso también fue significativa tras la realización de un partido $(z=-1,163 ; p=0,008)$. Se comprobó, tanto antes del partido $(p=0,011)$ como después $(p=0,008)$, que la distribución del peso no se ajustó a la distribución normal.
En cuanto al efecto del ejercicio sobre el porcentaje de agua, se siguió una dinámica similar, que no resultó estadísticamente significativa $(z=-1,178 ; p=0,239)$ tras el entrenamiento, ni tras la realización del partido $\left(t_{14}=-1,168 ; p=0,262\right)$.

Se evaluó la capacidad aeróbica de las deportistas $\left(\mathrm{VO}_{2 \text { máx }}\right)$ y la PSE como medidas del rendimiento atlético. $\mathrm{El} \mathrm{VO}_{2 \text { máx }}$ medio fue de $38,7 \pm 5,8 \mathrm{~mL} / \mathrm{kg} / \mathrm{min}$ (Tabla 5). Los resultados obtenidos en el Test de PSE mostraron que la media de PSE de cada jugadora el día la prueba física (después continuaron con el entrenamiento habitual) fue de dura a muy dura $(5,3 \pm 2,1$ puntos) y el día del partido de moderada a dura (3,8 $\pm 1,4$ puntos), según los valores de la escala de Borg del 0 al 10. Los valores de PSE correspondientes al entrenamiento no siguieron la distribución normal, según la Prueba de Shapiro-Wilk $(p=0,010)$. Al analizar si existían diferencias en la PSE entre el entrenamiento y el partido

Figura 1. Diferencias entre los requerimientos y la ingesta de las jugadoras.

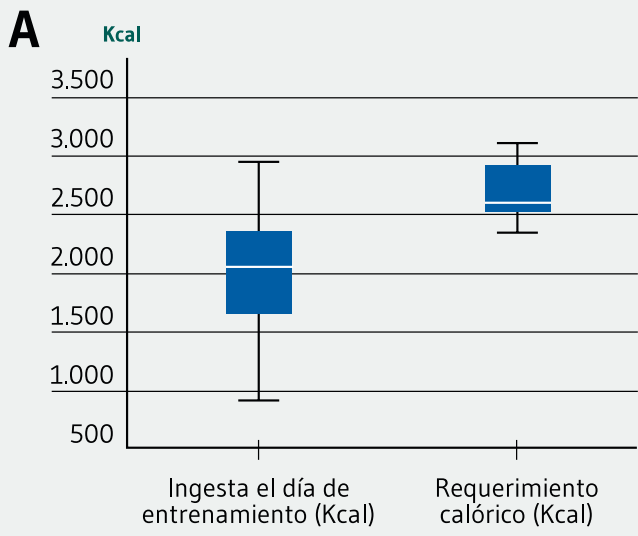

C Glúcidos (gr)

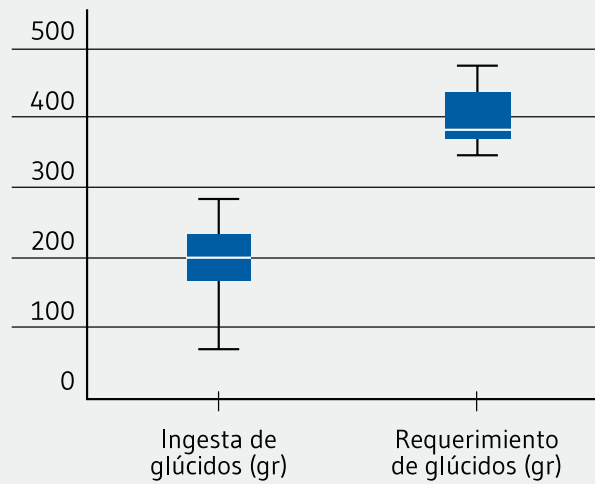

B Proteína (gr)

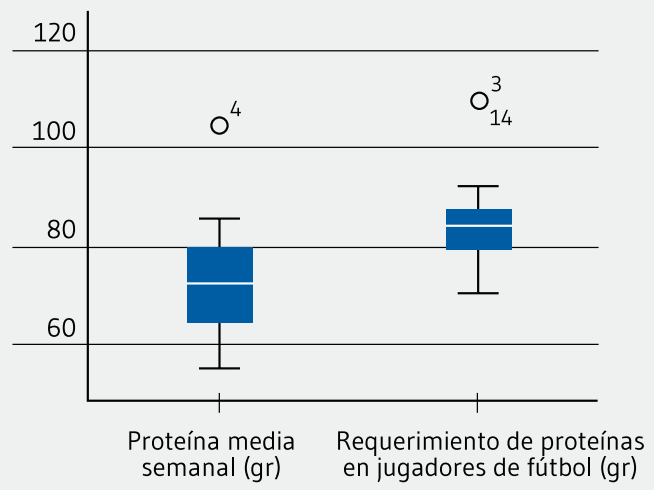

D Glúcidos (\%)

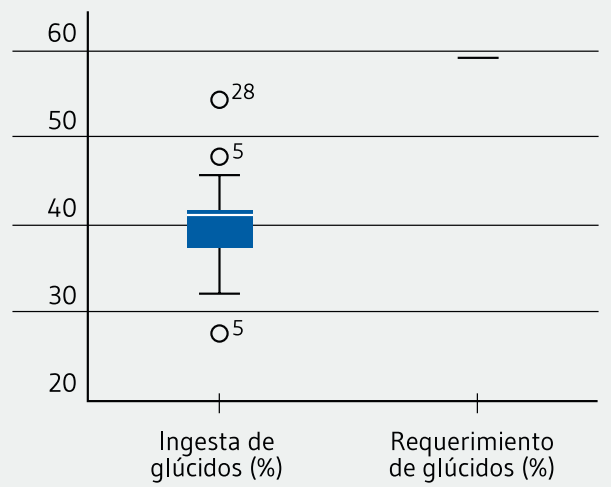

A: Diferencia entre la ingesta y el requerimiento calórico el día del entrenamiento; B: Diferencia entre la ingesta de proteína y el requerimientoen jugadores profesionales de fútbol; C: Diferencia entre la ingesta y el requerimiento de glúcidos (gr); D: Diferencia entre la ingesta y el requerimiento de glúcidos (\%). 
Tabla 4. Diferencia de peso y agua tras un entrenamiento o un partido.

\begin{tabular}{l|c|c|c|c|c|c|c|c} 
& & \multicolumn{3}{|c|}{ Antes del evento } & \multicolumn{3}{c}{ Después del evento } & \\
\hline Entrenamiento & N & M & Dt & SW & M & Dt & SW & p \\
Peso (kg) & 14 & 61,46 & 8,77 & 0,010 & 61,11 & 8,63 & 0,007 & $0,003^{\text {a }}$ \\
\% Agua & 13 & 56,91 & 4,31 & 0,046 & 57,28 & 4,29 & 0,254 & $0,239^{\text {a }}$ \\
Partido & N & M & Dt & SW & M & Dt & SW & p \\
\hline Peso (kg) & 15 & 61,46 & 8,72 & 0,011 & 60,94 & 8,63 & 0,010 & $0,008^{\text {a }}$ \\
\% Agua & 15 & 57,23 & 4,14 & 0,550 & 57,51 & 3,95 & 0,308 & $0,262^{\text {b }}$
\end{tabular}

$\mathbf{N}$ : frecuencia absoluta; M: media; Dt: desviación típica; SW: valor crítico de significación correspondiente al estadístico de contraste de la prueba de normalidad de Shapiro-Wilk; p: value correspondiente a la prueba aplicada para comprobar la diferencia de medias relacionadas; ${ }^{a}$ : prueba no paramétrica de rangos con signo de Wilcoxon; ${ }^{\text {: }}$ prueba t de Student para muestras relacionadas.

Tabla 5. Rendimiento deportivo según $\mathrm{VO}_{2 \text { Máx }}$ y prueba subjetiva de esfuerzo.

\begin{tabular}{l|c|c|c|c|c|c|c} 
Medida & $\mathbf{N}$ & $\mathbf{M}$ & $\mathbf{M n}$ & $\mathbf{D t}$ & Mín & Máx & SW \\
\hline VO $_{2 \text { Máx }}$ & 13 & 38,79 & 40,80 & 5,85 & 29,20 & 46,60 & 0,454 \\
PSE entrenamiento & 13 & 5,31 & 4,00 & 2,18 & 3,00 & 9,00 & 0,010 \\
PSE partido & 13 & 3,85 & 4,00 & 1,41 & 2,00 & 6,00 & 0,071
\end{tabular}

N: frecuencia absoluta; M: media; Mn: mediana; Dt: desviación típica; Mín: valor mínimo; Máx: valor máximo; SW: valor crítico de significación correspondiente al estadístico de contraste de la prueba de normalidad de Shapiro-Wilk

no se mostró un resultado estadísticamente significativo $(z=-1,490 ; p=0,136)$, a pesar de que los valores descriptivos apuntaban a que las futbolistas percibían que realizaban mayor esfuerzo en el entrenamiento $(M=5,31 ; D t=2,18)$ que en el partido $(M=3,85 ; D t=1,41)$.

Al estudiar la relación del rendimiento deportivo con la CC se obtuvo que la capacidad aeróbica $\left(\mathrm{VO}_{2 \operatorname{máx}}\right)$ se encontraba significativamente asociada al peso $(r=-0,595 ; p<0,05)$ en sentido inverso, lo que indicó que las deportistas con mayor peso tenían una capacidad aeróbica menor que las de un peso inferior (Tabla 6). También existió relación entre $\mathrm{VO}_{2 \text { máx }}$ e IMC $(r=-0,615 ; p<0,05)$, de manera que la mayor capacidad aeróbica se asoció con valores de IMC más bajos.

La PSE, tanto en el entrenamiento como en el partido, no se relacionó con la CC, a pesar de que el tamaño del efecto de algunas correlaciones fue de magnitud media. 
Tabla 6. Correlaciones de Pearson para estudiar la asociación de la composición corporal con el rendimiento deportivo.

\begin{tabular}{|c|c|c|c|c|}
\hline \multirow[b]{2}{*}{ Composición corporal } & & \multicolumn{3}{|c|}{ Rendimiento } \\
\hline & & $\mathrm{VO}_{2 \operatorname{Max}}$ & PSE entrenamiento & PSE partido \\
\hline \multirow{2}{*}{ Diferencia de peso tras entrenamiento $(\mathrm{kg})$} & $r$ & $-0,223$ & $-0,233$ & 0,053 \\
\hline & $\mathrm{n}$ & 13 & 13 & 12 \\
\hline \multirow{2}{*}{ Diferencia de agua tras el entrenamiento (\%) } & $r$ & $-0,158$ & 0,090 & 0,106 \\
\hline & $n$ & 12 & 12 & 12 \\
\hline \multirow{2}{*}{ Diferencia de peso tras el partido (kg) } & r & $-0,425$ & $-0,320$ & 0,070 \\
\hline & $n$ & 11 & 11 & 13 \\
\hline \multirow{2}{*}{ Diferencia de agua tras el partido (\%) } & r & $-0,196$ & $-0,108$ & 0,153 \\
\hline & $\mathrm{n}$ & 11 & 11 & 13 \\
\hline \multirow{2}{*}{ Peso (kg) } & r & $-0,595^{*}$ & $-0,235$ & $-0,310$ \\
\hline & $n$ & 13 & 13 & 13 \\
\hline \multirow{2}{*}{ Estatura $(\mathrm{cm})$} & r & 0,137 & $-0,040$ & 0,379 \\
\hline & $\mathrm{n}$ & 13 & 13 & 13 \\
\hline \multirow{2}{*}{ IMC (kg/m²) } & $r$ & $-0,615^{*}$ & $-0,288$ & $-0,338$ \\
\hline & $n$ & 13 & 13 & 13 \\
\hline \multirow{2}{*}{ Grasa (\%) } & $r$ & $-0,532$ & $-0,054$ & $-0,339$ \\
\hline & $n$ & 13 & 13 & 13 \\
\hline \multirow{2}{*}{ Masa muscular (kg) } & r & $-0,511$ & $-0,300$ & $-0,090$ \\
\hline & $n$ & 13 & 13 & 13 \\
\hline
\end{tabular}

r: coeficiente de correlación de Pearson; n: tamaño de la muestra. *p-value $<0,05$

\section{DISCUSIÓN}

El promedio de talla de las jugadoras $(165 \pm 6,7 \mathrm{~cm})$ está dentro de los valores medios encontrados en otros estudios similares 29,30 . No existen diferencias significativas entre la talla y la posición de juego, salvo la portera, que es de las jugadoras con mayor estatura, coincidiendo con otros autores ${ }^{13,31}$. El PC medio del $88,2 \%(61,4 \pm 8,7 \mathrm{~kg})$ es un PC adecuado para la media, sin embargo, en el $11,8 \%$ restante existe un PC excesivo ${ }^{13,30}$. Este exceso de PC puede suponer una desventaja en el campo, ya que el fútbol es un deporte en el que hay que mover el cuerpo con rapidez y agilidad ${ }^{11}$. EI IMC medio $\left(22,8 \pm 2,9 \mathrm{~kg} / \mathrm{m}^{2}\right)$ está dentro del rango que la OMS considera como normal para la población general $\left(18,5-24,9 \mathrm{~kg} / \mathrm{m}^{2}\right)^{32}$. Además, está dentro de los valores medios resultantes en otras mujeres futbolistas $\left(22,41 \pm 2,54 \mathrm{~kg} / \mathrm{m}^{2}\right)^{30}$, siendo únicamente superado por un $11,8 \%$ de las jugadoras, lo que coincide con las mismas participantes que presentan sobrepeso por un aumento de MG mayor de lo habitual en esta población. Al igual que ocurre con el PC, es destacable la amplia diferencia entre el valor mínimo y el máximo, con un rango de $10,1 \mathrm{~kg} / \mathrm{m}^{2}$ para el IMC y de $29 \mathrm{~kg}$ para el PC. En relación al porcentaje de MG, si comparamos los porcentajes de grasa obtenidos $(24,5 \pm 5,5 \%)$ con los porcentajes encontrados en un equipo de fútbol femenino que juega en Primera División Nacional
$(21,9 \pm 0,9 \%)^{13}$, las participantes presentaron un promedio de porcentaje MG elevado. Sin embargo, si lo referenciamos con los porcentajes resultantes de un equipo de fútbol femenino que juega en la Primera División Regional $(29,4 \pm 1,4 \%)^{13}$, con una frecuencia de entrenamiento de 4,5 horas semanales, igual al de las jugadoras del estudio, sólo un $11,8 \%$ de la muestra supera este porcentaje. No obstante, los diversos estudios realizados sugieren que por término medio las mujeres futbolistas presentan en torno a un $21 \%$ de $\mathrm{MG}^{13}$, siendo superado este porcentaje por el $64,7 \%$ de la muestra. El alto grado de adiposidad puede alterar el rendimiento deportivo de las mismas, ya que el porcentaje de grasa es el componente más influyente en el rendimiento deportivo, habiendo una relación inversa entre la cantidad de GC y el rendimiento ${ }^{12,18}$. No obstante, un porcentaje de MG muy bajo, tampoco permite obtener un buen estado de salud y un buen rendimiento físico, siendo necesario en la mujer, un mínimo de un $12 \%$ de MG $^{12}$, presentando todas las jugadoras un porcentaje superior a este valor.

Destacar que tan sólo un $25 \%$ de las jugadoras se aproximaba a las recomendaciones de ingesta calórica. Esto es importante, pues una ingesta insuficiente de energía, también puede afectar al rendimiento deportivo, ya que la falta de sustrato energético (glucógeno, ácidos grasos) es una causa de fatiga $5,8,27,33$. Además, es destacable que un $18,8 \%$ de las jugadoras realiza una ingesta calórica por debajo de $30 \mathrm{kcal} / \mathrm{kg}$ de PC magro al día. Esto, además de empeorar 
el rendimiento deportivo, puede tener consecuencias negativas para el metabolismo, la función menstrual y para la reabsorción y formación ósea27.

El aporte energético de las jugadoras tampoco es el adecuado en cuanto a macronutrientes y distribución de los mismos, ya que a través de los registros dietéticos se observa cómo muchas de las futbolistas realizan únicamente 2 ingestas el día de entrenamiento y/o partido, siendo además estas comidas bajas en HC. El perfil calórico de las jugadoras

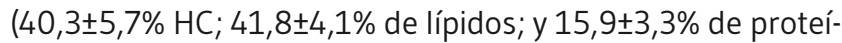
nas), se aleja del perfil deseable (55-60\% HC, 25-30\% de lípidos y $12-15 \%$ de proteínas), teniendo en cuenta la fisiología de este deporte, así como el mantenimiento de la salud y el rendimiento deportivo $34,35,36$. En relación al porcentaje de HC medio consumido (40,3 $35,6 \%$ ), señalar que sólo un $5,8 \%$ de las jugadoras consumió más del $50 \%$ de la energía total a partir de $\mathrm{HC}$ y, ninguna supera el $55 \%$. Por otra parte, si se especifica el tipo de HC, se puede destacar cómo el $100 \%$ de las jugadoras presentan una ingesta de $\mathrm{HC}$ simples de $18,4 \pm 3,6 \%$, correspondientes a $87,97 \pm 26,89 \mathrm{~g} / \mathrm{día}$, superior a la recomendación $(<5 \% \text { de las } \mathrm{kcal} \text { totales de la dieta })^{37}$. Estos valores son el resultado de un consumo excesivo de bebidas carbonatadas azucaradas, bollería industrial, golosinas, etc. El consumo de este tipo de alimentos y bebidas es realizado, por muchas de ellas, momentos previos a la práctica de ejercicio, pudiendo provocar una "hipoglucemia reactiva" y una elevada liberación de insulina,35,38. Por otra parte, al analizar la cantidad de $\mathrm{HC}$ que consumen horas previas (6 horas antes) al entrenamiento o partido, se extrae que el $50 \%$ de las jugadoras no realizan una ingesta de $\mathrm{HC}$ suficiente el día de entrenamiento y un 31,3\% tampoco el día de partido. No consumir una cantidad suficiente de $\mathrm{HC}$, no permite conseguir unos niveles de glucógeno adecuados, agotándose antes dicho sustrato y apareciendo antes la fatiga $6,35,38$.

En cuanto a las proteínas, el 50\% de las jugadoras no cumple las recomendaciones de ingesta, por exceso o por defecto, para este deporte $(1,2-1,6 \mathrm{~g} / \mathrm{kg} / \mathrm{día})^{39}$, siendo un dato que evidencia que la alimentación de las jugadoras no es adecuada. No tener un consumo adecuado de proteínas puede dificultar el crecimiento muscular, la reparación y la adaptación que sobreviene después del ejercicio ${ }^{6,8,39}$. Con respecto a las grasas, presentan una ingesta $(41,8 \pm 4,1 \%)$ por encima del porcentaje recomendado ${ }^{8}$, con un perfil de lípidos inadecuado, teniendo todas las futbolistas de la

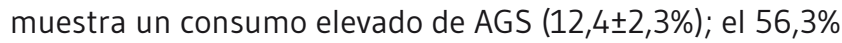
de las jugadoras alto de AGM $(21 \pm 2,4 \%)$ y un $50 \%$ bajo de $\operatorname{AGP}(5,4 \pm 1,0 \%)$. Es destacable, que aunque en su conjunto, el porcentaje calórico derivado del consumo de alcohol no es significativo $(6,5 \pm 5,2 \%)$, un $6,25 \%$ de las jugadoras tiene un aporte calórico derivado del mismo por encima al $10 \%$ de las calorías totales, superando las recomendaciones ${ }^{38}$. Al analizar otros micronutrientes, se obtiene que el promedio de ingesta de hierro de las jugadoras $(12,5 \pm 3,12 \mathrm{mg} /$ día) está por debajo de las cantidades diarias recomendadas de hierro para mujeres de entre 19 a 50 años $(18 \mathrm{mg} / \text { día })^{40}$. Por otra parte, es importante destacar que un $12,5 \%$ de las jugadoras notificó presentar anemia ferropénica, observando en éstas, al analizar su ingesta, que no tienen un aporte suficiente de hierro a través de su alimentación. Es frecuente encontrar una elevada prevalencia de anemia ferropénica en deportistas, especialmente en mujeres ${ }^{41}$, siendo el aporte insuficiente de hierro a través de la dieta una de las principales causas, aunque no la única ${ }^{42}$. La deficiencia de hierro puede tener como consecuencia una alteración en el rendimiento físico de las deportistas, debido al papel importante que juega el hierro en el transporte y liberación de oxígeno a la célula muscular y en la obtención de energía en el metabolismo aeróbico ${ }^{41}$

Basándonos en las últimas recomendaciones en el patrón de la Dieta Mediterránea 22 , los datos resultantes del KIDMED muestran que las jugadoras tienen una baja ingesta de verduras, hortalizas y frutas (un $53 \%$ no consume al menos una ración de verdura/hortaliza al día, mismo porcentaje que no consume una segunda pieza de fruta), teniendo en cuenta que se deben consumir al menos 2 raciones de verdura/ hortaliza al día y 3 piezas de fruta para obtener un correcto aporte de agua, vitaminas, minerales y fibra. El consumo de cereales, arroz y pasta también es bajo (el 53\% no los consume 5 o más veces por semana), debiendo incluir 1-2 raciones de estos alimentos en las comidas principales. El consumo de pescado tampoco es el adecuado (el $53 \%$ no toma pescado 3-4 veces por semana) y el consumo recomendado de legumbres (al menos 2 veces por semana) no es realizado por el $29 \%$ de las jugadoras, siendo este último alimento una fuente importante de $\mathrm{HC}$ complejos junto con los cereales, arroz, pasta y tubérculos. La ingesta óptima de lácteos y derivados (2 raciones/día) no es realizada por un $44 \%$. Los lácteos son la mejor fuente de calcio, siendo este micronutriente esencial para la constitución de la densidad y la masa ósea ${ }^{1,6}$. Un $12 \%$ no realiza el desayuno y, de quienes lo hacen (88\%), un 29\% opta por bollería industrial. Estos alimentos son ricos en azúcares sencillos y en grasas hidrogenadas, elevan rápidamente los niveles de glucosa, disparando la producción de insulina ${ }^{34}$. Además, el $88 \%$ de las futbolistas realiza alguna comida en un establecimiento de comida rápida, siendo éstos últimos característicos por ofrecer alimentos con una alta densidad calórica, produciendo un desequilibrio energético y nutricional en la dieta. Por otra parte, el número de comidas que realizan al día no se acerca a la recomendación (sólo un 12,5\% realiza más de 4 ingestas). No repartir la ingesta de alimentos en varias 
comidas a lo largo del día (4-5) puede producir un reparto inadecuado de energía, haciendo que la carga de glucógeno sea menos eficiente ${ }^{4}$. Por último, destacar que hay un porcentaje de jugadoras (18\%) que no usa de manera habitual el aceite de oliva, pudiendo ser una de las causas de que el perfil lipídico se aleje de los objetivos nutricionales ${ }^{35}$.

La pérdida de peso observada el día de entrenamiento $(0,6 \pm 3,2 \%$ de $P C)$ y el día de partido $(1,1 \pm 5,2 \%$ de $P C)$, podría suponer una ligera deshidratación por la pérdida de agua, ya que aunque hay que tener en cuenta las pérdidas derivadas del consumo de glucógeno y de las oxidaciones de los principios inmediatos, el $71,4 \%$ tuvo una pérdida superior o igual al $1 \%$ el día de entrenamiento y un $64,3 \%$ el día de partido (Tabla 4). Estas pérdidas (entre 1-2\%), son suficientes para producir una elevación de la temperatura corporal durante el ejercicio y disminuir el rendimiento físico ${ }^{43}$.

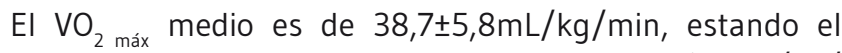
$41,7 \%$ de las jugadoras por debajo de la media $(39 \mathrm{~mL} / \mathrm{kg} /$ min) de futbolistas de élite de primera división ${ }^{31}$. Si tenemos en cuenta otros estudios ${ }^{19}$, el $100 \%$ presenta un $\mathrm{VO}_{2 \text { máx }}$ bajo y sólo un 46,2\% presenta una capacidad aeróbica buena según los valores de referencia de García Manso y Cols. ${ }^{44}$. No obstante, sería preciso calcular el $\mathrm{VO}_{2 \text { máx }}$ a través de otros métodos de evaluación (prueba de esfuerzo, monitores de frecuencia cardiaca o similares) para tener un valor más confiable y válido.

Las puntuaciones obtenidas en el test de PSE, son mayores el día de entrenamiento $(5,3 \pm 2,1)$ que el día de partido $(3,8 \pm 1,4)$. Esto puede deberse a que la intensidad de ejercicio fue mayor el día de entrenamiento, ya que previamente se realizó el tes CN. Destacar que las jugadoras que mayor $\mathrm{VO}_{2 \text { máx }}$ presentan son las que mayor nivel de esfuerzo dicen soportar a través de la puntuación en la escala de Borg ${ }^{20,22}$, existiendo una relación directa entre ambos parámetros.

\section{Limitaciones en el estudio:}

- El cuestionario KIDMED fue diseñado y validado en 2004, para valorar la adherencia de la población española infanto-juvenil a la Dieta Mediterránea de 2 a 24 años de edad, saliendo una de las participantes de la muestra de estas edades (36 años).

- Dentro del protocolo de buenas prácticas de la BIA, se recomienda que en mujeres se efectúe la medición lejos del período premenstrual para evitar la retención líquido, factor que no se tuvo en cuenta, pues las sesiones de intervención no se pudieron cambiar de fecha (adelantar o retrasar).

\section{CONCLUSIONES}

El déficit de adecuación a la dieta es mayor de lo esperado para un equipo semiprofesional que se entrena semanalmente para competir, sólo el 5,9\% de las atletas mantiene una adherencia a la Dieta Mediterránea óptima. La ingesta de nutrientes por parte de las jugadoras no corresponde con sus requerimientos, ni en macronutrientes ni en hierro. En porcentaje de MG del conjunto estudiado está en consonancia con los valores estándares de referencia, salvo 2 jugadoras en las que existe un porcentaje de MG por encima de dichos valores. Se ha encontrado una relación inversa entre IMC y peso, y los valores de $\mathrm{VO}_{2 \text { máx. También se ha }}$ observado una relación inversa entre la cantidad de GC y el rendimiento deportivo, pudiendo verse alterado éste último en las jugadoras estudiadas. Debido a la importancia que la nutrición juega en la competición y el rendimiento deportivo, creemos fundamental trabajar para aplicar el beneficio de una nutrición óptima y personalizada en el entorno del fútbol femenino.

\section{AGRADECIMIENTOS}

Agradecer a todo el quipo Femenino de Torrelodones C.F. y a sus entrenadores.

\section{CONFLICTO DE INTERESES}

Los autores manifiestan que no existe conflicto de intereses al redactar el manuscrito.

\section{BIBLIOGRAFÍA}

1. Grimm K, Kirkendall D. Salud y buena condición física de las futbolistas. 1a ed. FIFA, editor. Altstätten: rva Druck und Medien AG; 2007.

2. Ruiz F, Gravina L, Gil J, Díaz E, Gil S, Hoyos I. Ingesta de energía y macronutrientes en jóvenes deportistas de diferentes edades. Osasunaz. 2007; 233-47.

3. Martínez C, Sánchez P. Estudio nutricional de un equipo de fútbol de tercera división. Nutr Hosp. 2013; 28(2): 319-24.

4. Gutiérrez F, Canda A, Heras ME, Boraita A, Rabadán M, Lillo P, et al. Análisis, valoración y monitorización del entrenamiento de alto rendimiento deportivo. 1aㅡ ed. CSD, editor. Madrid: Imprenta Nacional del BOE; 2010. 
5. González-Gross M, Gutiérrez A, Mesa JL, Ruiz-Ruiz J, Castillo MJ. La nutrición en la práctica deportiva: adaptación de la pirámide nutricional a las características de la dieta del deportista. Arch Latinoam Nutr. 2001; 51(4): 321-31.

6. Maughan R, Burke L, Kirkendall D. F-MARC - Nutrición para el fútbol. $1^{\underline{a}}$ ed. FIFA, editor. Altstätten: rva Druck und Medien AG; 2005

7. Garret WE, Kirkendall DT, Contiguglia SR. Medicina del fútbol. 1a ed. Barcelona: Paidotribo; 2005.

8. American College of Sports Medicine, American Dietetic Association, Dietitians of Canada. Joint Position Statement: nutrition and athletic performance. American College of Sports Medicine, American Dietetic Association, and Dietitians of Canada. Med Sci Sports Exerc. 2000; 32(12): 2130-45.

9. Maughan RJ, Shirreffs SM. Nutrition and hydration concerns of the female football player. Br ] Sports Med. 2007; 41(Suppl 1): i60-3.

10. Palacios N, Montalvo Z, Ribas AM. Alimentación, nutrición e hidratación en el deporte. CSD, editor. Madrid: Imprenta Nacional del BOE; 2009.

11. Varela G, Silvestre D. Nutrición, vida activa y deporte. 1a ed. Instituto Tomás Pascual Sanz, Universidad San Pablo CEU, editores. Madrid: IM\& C; 2010.

12. Pérez-Guisado J. Rendimiento deportivo: composición corporal, peso, energía-macronutrientes y digestión (I). Arch Med Deporte. 2009; 26(133): 389-94.

13. Sedano S, Cuadrado G, Redondo JC, de Benito A. Perfil antropométrico de las mujeres futbolistas españolas. Análisis en función del nivel competitivo y de la posición ocupada habitualmente en el terreno de juego. Apunts. 2009; 98(4): 78-87.

14. Polman R, Walsh D, Bloomfield J, Nesti M. Effective conditioning of female soccer players. J Sports Sci. 2004; 22(2): 191-203.

15. Mladenović I. Developing characteristics and functional abilities of top female football players. Facta Univ Ser Med Biol. 2005; 12(2): 97-9.

16. Hewitt A, Norton K, Lyons K. Movement profiles of elite women soccer players during international matches and the effect of opposition's team ranking. J Sports Sci. 2014; 32(20): 1874-80.

17. Bradley PS, Dellal A, Mohr M, Castellano J, Wilkie A. Gender differences in match performance characteristics of soccer players competing in the UEFA Champions League. Hum Mov Sci. 2014; 33: 159-71.

18. Wilmore JH, Costill DL. Fisiología del esfuerzo y del deporte. $2 \mathrm{a}$ ed. Barcelona: Paidotribo; 2007.

19. Herrero A. Cienantropometría: Composición corporal y somatotipo de futbolistas que desarrollan su actividad física en equipos de la comunidad autónoma de Madrid. Arch Med Deporte. 2007; 24(117): 65-9.

20. Bahamondes C, Cifuentes BM, Lara E, Bernal FJ. Composición Corporal y Somatotipo en Fútbol Femenino: Campeonato Sudamericano Sub-17. Int J Morphol. 2012; 30(2): 450-60.

21. Almagià $A A$, Rodríguez $F$, Barrraza FO, Lizana PJ, Jorquera CA. Perfil antropométrico de jugadoras chilenas de fútbol femenino. Int J Morphol. 2008; 26(4): 817-21.

22. Serra-Majem L, Ribas L, Ngo J, Ortega RM, García A, PérezRodrigo $C$, et al. Food, youth and the Mediterranean diet in Spain. Development of KIDMED, Mediterranean Diet Quality Index in children and adolescents. Public Health Nutr. 2004; 7(7): 931-5.

23. Ortega RM, López-Sobaler AM, Requejo P. La composición de los alimentos: herramienta básica para la valoración nutricional. 1a ed. Madrid: Editorial Complutense; 2004.

24. Barbero JC, Barbero V. Relación entre el consumo máximo de oxígeno y la capacidad para realizar ejercicio intermitente de alta intensidad en jugadores de fútbol sala. Futsalcoach [revista en internet]. 2006 [consulta: 12/05/2014].
Disponible en: http://futsalcoach.es/web v2/area tecnica/ archivos/449_relacionconsumooxigeno_priñt.pdf

25. Casamichana D, Castellano ], Blanco-Villaseñor Á. Estudio de la percepción subjetiva del esfuerzo en tareas de entrenamiento en fútbol a través de la teoría de la generalizabilidad. Rev Psicol Deporte. 2012; 21(1): 35-40.

26. Burkhalter N. Evaluación de la escala Borg de esfuerzo percibido aplicada a la rehabilitación cardiaca. Rev Lat Am Enfermagem. 1996; 4(3): 65-73.

27. Burke L. Nutrición en el deporte: un enfoque práctico. $1^{\text {a }}$ ed. Madrid: Editorial Médica Panamericana; 2009.

28. Food and Agriculture Organization. Composición corporal, funciones de los alimentos, metabolismo y energía. [Citado el 30 de Marzo del 2015]. Disponible en: http://www.fao.org/ docrep/006/w0073s/w0073s0c.

29. González M, Pérez J, Garrido R, Garnés AF. Composición corporal de los futbolistas de equipos alicantinos. Seleccion. 2004; 13(4): 155-63.

30. Gómez M, Barriopedro Ml. Características fisiológicas de jugadoras españolas de fútbol femenino. Kronos [revista en internet]. 2005 [consulta: 12/05/2014];3(7):27-32. Disponible en: http://www.revistakronos.com/kronos/index. php?articulo $=50$

31. Herrero de Lucas A, Cabañas MD, Maestre I. Morfotipo del futbolista profesional de la Comunidad Autónoma de Madrid. Composición corporal. 2004 [consulta: 12/05/2014];12(1):72-Disponible en: http://upcommons.upc. edu/revistes/handle/2099/6822? locale=es

32. OMS. Obesidad y sobrepeso [internet]. Génova: WHO Media Center; 2006 [actualizado mayo 2012; citado 16/06/2013]. Disponible en: http://www.who.int/mediacentre/factsheets/ fs $311 /$ es/

33. Gusi N. Efectos biomecánicos de la fatiga. Apunts. 1991; 26(4): 43-50

34. González JA, Cobos I, Molina E. Estrategias nutricionales para la competición en el fútbol. Rev Chil Nutr. 2010; 37(1): 118-22.

35. Arecheta C, Gómez M, Lucía A. La Importancia del VO para realizar esfuerzos intermitentes de alta intensidad en el fútbol femenino de élite. Kronos [revista en internet]. 2006 [consulta: 12/05/2014];5(9):4-12. Disponible en: http://www. revistakronos.com/kronos/index.php?articulo=71

36. Leblanc JC, Le Gall F, Grandjean V, Verger P. Nutritional intake of French soccer players at the clairefontaine training center. Int J Sport Nutr Exerc Metab. 2002; 12(3): 268-80.

37. WHO. WHO opens public consultation on draft sugars guideline [internet]. Génova: WHO Media Center; 2014 [actualizado marzo 2014; citado 20/05/2014]. Disponible en: http://www. who.int/mediacentre/news/notes/2014/consultation-sugarguideline/en/

38. Lizarraga A, Barbany JR, Pons V, Pasabán E, Capdevila L. Alimentación y deporte: tendencias actuales, tecnología innovación y pedagogía. Instituto Tomás Pascual Sanz, editor. Madrid: IM\&C; 2010

39. Palacios N. Vida activa, ejercicio y alimentación. En: Fundación Española de la Nutrición, editor. Libro blanco de la nutrición en España. Madrid: Lesinguer; 2013. P. 551-9.

40. Sociedad Española de Dietética y Ciencias de la Alimentación. Ingesta Recomendada de Nutrientes (I.R.) ó R.D.A. Recomendaciones de ingesta de nutrientes para la población española [internet]. Madrid: Sociedad Española de Dietética y Ciencias de la Alimentación; 2012 [citado 12/05/2014]. Disponible en: http://www.nutricion.org/recursos_y_ utilidades/necesid nutrientes.htm

41. Legaz A. Atletismo ēspañol: análisis básico de la pseudoanemia, anemia ferropénica y anemia megaloblástica. Rev Int Med Cienc Act Fís Deporte [revista en internet]. 2000 [consulta: 12/05/2014];1(1):65-83. Disponible en: http://cdeporte. 
Rev Esp Nutr Hum Diet. 2015; 19(1): 36 - 48

Valoración nutricional, evaluación de la composición corporal y su relación con el rendimiento deportivo en un equipo de fútbol femenino

rediris.es/revista/revista1/anemia1.htm

42. Comunidad de Madrid. Nutrición deportiva [internet]. Madrid: Comunidad de Madrid [citado 20/05/2014]. Disponible en: http://www.madrid.org/cs/Satellite?c=CM_InfPractica_FA\&ci $\mathrm{d}=1142628793327 \&$ language $=$ es\&pagenäme $=$ ComunidadM adrid\%2FEstructura\&pv=1142652499617
43. Martínez JR, Villarino AL, Polanco I, Iglesias C, Gil P, Ramos $P$, et al. Recomendaciones de bebida e hidratación para la población española. Nutr Clin Diet Hosp. 2008; 28(2): 3-19.

44. García JM.; Navarro M, Ruiz JA. Pruebas para la valoración de la capacidad motriz en el deporte: evaluación de la condición física. 1aㅡ ed. Grada, Gymnos, editores. Madrid: Gymnos; 1996. 